\title{
TNF- $\alpha$ gene expression is increased following zinc supplementation in type 2 diabetes mellitus
}

\author{
Anna Chu • Meika Foster • Dale Hancock • \\ Kim Bell-Anderson • Peter Petocz • Samir Samman
}

Received: 5 September 2014/ Accepted: 28 October 2014/Published online: 15 November 2014

(C) Springer-Verlag Berlin Heidelberg 2014

\begin{abstract}
Chronic low-grade inflammation in type 2 diabetes mellitus (DM) can elicit changes in whole-body zinc metabolism. The interaction among the expression of inflammatory cytokines, zinc transporter and metallothionein (MT) genes in peripheral blood mononuclear cells in type 2 DM remains unclear. In a 12-week randomized controlled trial, the effects of zinc (40 mg/day) supplementation on the gene expression of cytokines, zinc transporters and MT in women with type 2 DM were examined. In the zinc-supplemented group, gene expression of tumour necrosis factor $(T N F)-\alpha$ tended to be upregulated by $27 \pm 10 \%$ at week 12 compared to baseline $(P=0.053)$. TNF- $\alpha$ fold change in the zinc-treated group was higher than in those without zinc supplementation $(P<0.05)$. No significant changes were observed in the expression or fold change of interleukin $(I L)-1 \beta$ or $I L-6$. Numerous bivariate relationships were observed between
\end{abstract}

Electronic supplementary material The online version of this article (doi:10.1007/s12263-014-0440-4) contains supplementary material, which is available to authorized users.

A. Chu $\cdot$ M. Foster $\cdot$ K. Bell-Anderson $\cdot$ S. Samman Discipline of Nutrition and Metabolism, School of Molecular

Bioscience, University of Sydney, Sydney, NSW 2006, Australia

D. Hancock

Discipline of Molecular Biology, School of Molecular

Bioscience, University of Sydney, Sydney, NSW 2006, Australia

P. Petocz

Department of Statistics, Macquarie University, Sydney,

NSW 2109, Australia

S. Samman $(\bowtie)$

Department of Human Nutrition, University of Otago,

PO Box 56, Dunedin 9054, New Zealand

e-mail: samir.samman@otago.ac.nz the fold changes of cytokines and zinc transporters, including $Z n T 7$ with $I L-1 \beta(P<0.01), I L-6(P<0.01)$ and $T N F-\alpha(P<0.01)$. In multiple regression analysis, $I L-1 \beta$ expression was predicted by the expression of all zinc transporters and MT measured at baseline $\left(r^{2}=0.495\right.$, $P<0.05)$ and at week $12\left(r^{2}=0.532, P<0.03\right)$. The current study presents preliminary evidence that zinc supplementation increases cytokine gene expression in type 2 DM. The relationships found among zinc transporters, MT and cytokines suggest close interactions between zinc homeostasis and inflammation.

Keywords Inflammation - Cytokines - Zinc transporters · Metallothionein - Gene expression - Type 2 diabetes mellitus

\section{Introduction}

Zinc is involved in the biosynthesis, storage and secretion of insulin within the pancreatic $\beta$-cells (Huang 2014). Furthermore, intracellular zinc can act directly on the insulin signalling pathway to improve glucose uptake and insulin sensitivity in peripheral tissues (Haase and Maret 2005; Foster and Samman 2010). Individuals with type 2 diabetes mellitus (DM) are suggested to be of poor zinc status due to the presentation of higher urinary zinc excretion and lower serum zinc concentrations (Jansen et al. 2009). Suboptimal zinc status can affect glycaemic control by compromising the production and secretion of insulin in the pancreas (Huber and Gershoff 1973) and impacting insulin sensitivity in peripheral tissues (Jansen et al. 2009; Kelleher et al. 2011). Hence, the persistent elevation of blood glucose concentration featured in type 2 DM may be attributed partly to perturbed zinc homeostasis. 
Cellular zinc homeostasis is regulated primarily by two families of zinc transporters and metallothionein (MT) (Lichten and Cousins 2009). The Zrt- and Irt-like protein (Zip) (SLC39) family of transporters is responsible for increasing cytoplasmic zinc concentration by transporting zinc from intracellular organelles or the extracellular space. Conversely, the ZnT (SLC30) family of transporters functions to decrease the cytoplasmic zinc concentration by transporting zinc from the cytosol into the extracellular space or internal organelles, such as those involved in secretory pathways. MT acts as a target for zinc ion binding in the cytoplasm and is believed to assist in the trafficking of zinc ions through the cell (Babula et al. 2012). The regulatory control of cellular zinc transporters and MT is complex and has been shown to be influenced by zinc status, systemic glucose and inflammatory cytokines (Lichten and Cousins 2009; Hennigar and Kelleher 2012).

Cellular zinc transporters in different cell types have been shown to be both up- and downregulated to meet the changing demand for zinc in inflammatory conditions (Foster and Samman 2012). The redistribution of zinc from the systemic circulation into cellular compartments is thought to be crucial for immune function. During acute infection, an increase in systemic interleukin (IL)-6 can induce hepatic accumulation of zinc, which contributes to the rapid decrease in the plasma zinc concentration often seen in the acute phase response (Liuzzi et al. 2005). The chronically activated innate immune system in type $2 \mathrm{DM}$ (Pickup 2004) also can elicit changes to whole-body zinc metabolism. Exposure to systemic cytokines, such as IL$1 \beta$, have been associated with downregulation of the zinc transporters shown to play a role in insulin production and storage in the pancreatic $\beta$-cells (Egefjord et al. 2009).

The expressions of immune markers, such as IL-1 $\beta$, IL6 and TNF- $\alpha$, often are linked to the progression of type 2 DM (Donath and Shoelson 2011). Specifically, increase in IL-1 $\beta$ has been shown to promote pancreatic $\beta$-cell destruction (Banerjee and Saxena 2012) which, in combination with TNF- $\alpha$ and IL-6, may synergistically exacerbate the extent of $\beta$-cell apoptosis and disease progression (Cnop et al. 2005). Furthermore, TNF- $\alpha$ and IL-6 can promote insulin resistance in peripheral tissues by modulating the expression of key regulators in the insulin signalling pathway (Mlinar et al. 2007). Attenuation of CRP and inflammatory cytokine production may be achieved by dietary supplementation of anti-inflammatory nutrients, such as zinc and n-3 polyunsaturated fatty acids (PUFA) (Calder 2006; Foster and Samman 2012). In a 6-week cross-over intervention study which manipulated the composition of fatty acids, a diet high in $\alpha$-linolenic acid (ALA) derived from FSO resulted in significant reductions in cytokine production and vascular inflammation in hypercholesterolemic subjects (Zhao et al. 2004, 2007).
The effectiveness of a modest level of ALA supplementation on inflammation in type 2 DM is currently unknown.

Similarly, the effect of zinc supplementation on inflammatory biomarkers in type 2 DM remains largely unexplored. While zinc supplementation appears to reduce the level of systemic CRP and IL-6 (Bao and Prasad 2010), conflicting findings are reported for the effect of zinc supplementation on ex vivo cytokine production in stimulated mononuclear cells (Aydemir et al. 2006; Prasad and Beck 2007). In a recent zinc supplementation trial in individuals with type 2 DM (Foster et al. 2013b), we reported that systemic inflammatory markers were associated with the gene expression of zinc transporters and MT, suggesting an interplay between systemic markers of inflammation and cellular zinc transport. Consistent with our observation, a recent study in obese women showed levels of systemic C-reactive protein (CRP) and tumour necrosis factor (TNF)$\alpha$ to be inversely correlated with a range of zinc transporter gene expressions (Noh et al. 2014).

Peripheral blood mononuclear cells (PBMC) have been used as a candidate target tissue to detect transcriptome changes in response to dietary modification in humans (de Mello et al. 2012). The expression of zinc transporters and MT in PBMC have been described previously in a healthy population (Foster et al. 2011) and those with type 2 DM (Foster et al. 2013b). However, the interactions between the gene expression of inflammatory cytokines, zinc transporters and MT remain unclear. To extend our previous report, specifically the relationships between systemic cytokines (IL-1 $\beta$, IL-6 and TNF- $\alpha$ ) and zinc transporters and MT (Foster et al. 2013b), the gene expressions of $I L-1 \beta, I L-6$ and $T N F-\alpha$ in PBMC were measured to provide further insight into the interactions between zinc and the immune system in type 2 DM. The present study aims to investigate the effect of zinc on the gene expression of inflammatory cytokines in PBMC and explore possible relationships between the gene expression of cytokines, zinc transporters and MT.

\section{Materials and methods}

\section{Study design}

The study design has been described previously (Foster et al. 2013a, b). In brief, 48 participants were enrolled in a randomized, double-blind, placebo-controlled trial. The primary inclusion criteria were that participants be postmenopausal ( $>12$ months without menses), have normal glomerular filtration rate and microalbumin/creatinine ratio, and were diagnosed with type $2 \mathrm{DM}$, controlled by either diet and lifestyle or oral hypoglycaemic medication. Postmenopausal women were chosen for this trial as they have been identified as an understudied population group, 
and the inclusion of women who suffer from chronic conditions such as type 2 DM contributes biomedical knowledge that advances patient care (Kim et al. 2010). Enrolled participants were randomized into four equal groups to receive a total of $40 \mathrm{mg} /$ day elemental zinc (' $\mathrm{Zn}$ Group'), 2,000 mg/day flaxseed oil ('FSO group'), both zinc and flaxseed oil ('Zn + FSO group'), or placebo for 12 weeks. Placebo capsules that were identical in appearance to their active counterparts were given to the placebo groups. Zinc placebo capsules contained cellulose, while olive oil was used as the placebo for FSO. All procedures followed were in accordance with the ethical standards of the Human Research Ethics Committee of the University of Sydney. Informed consent was obtained from all participants for being included in the study. The study protocol was registered at www.clinicaltrials.gov (NCT01505803).

Markers of systemic inflammation, glycaemia and zinc

Venous blood samples from participants were collected at baseline, and at weeks 4,8 and 12 for the analysis of glucose, haemoglobin A1c (HbA1c), cytokines, CRP and zinc. Serum glucose was measured by glucose hexokinase UV method using the Gluco-quant reagent kit adapted for a Modular PPE auto-analyser (Roche Diganostics, Basel, Switzerland). Serum insulin was determined by chemiluminescent microparticle immunoassay on an Architect i2000SR Analyzer (Abbott Laboratories, Abbott Park, IL, USA). HbA1c was assayed using ion-exchange high performance liquid chromatography (HPLC) on a Variant II analyser equipped with the Variant II NU Program (BioRad Laboratories, Hercules, CA, USA), according to the manufacturer's protocol. Plasma zinc was determined using inductively coupled plasma mass spectrometry (Agilent 7500ce ICPMS, Santa Clara, CA, USA). The human cytokine/chemokine Milliplex MAP kit (Millipore, Billerica, MA, USA) was used for the simultaneous quantification of serum IL-1 $\beta$, IL- 6 and TNF- $\alpha$ concentrations, according to the manufacturer's instructions. Samples were analysed on a Luminex 100 Bioanalyser (Luminex Corp., Austin, TX, USA) using Fidis multiplex technology (Biomedical Diagnostics, Marne la Vallée, France). Serum CRP was measured using the Tina-quant CRP (gen.3) immunoturbidimetric method adapted for a Roche Modular PPE analyser (Roche Diagnostics, Basel, Switzerland) according to the manufacturer's instructions.

Zinc transporter, metallothionein and cytokine gene expressions

PBMC were isolated from blood samples collected at baseline and week 12, processed through to cDNA and stored at $-80{ }^{\circ} \mathrm{C}$ until quantitative real-time PCR analysis.
Unstimulated PBMC from individual samples were extracted, and total RNA was prepared using the RNAqueous Small Scale Phenol-Free Total RNA Isolation Kit (Applied Biosystems-Life Technologies Australia Pty Ltd, Victoria, Australia) according to the manufacturer's instructions. Total RNA was reverse transcribed into cDNA using the Superscript VILO cDNA Synthesis System (Invitrogen-Life Technologies Australia Pty Ltd, Victoria, Australia) following the manufacturer's protocol. Forty complete samples of cDNA from study participants were recovered for cytokine gene expression analysis. Inventoried TaqMan gene expression assays were obtained for $I L$ $1 \beta, I L-6$ and $T N F-\alpha$ for relative quantification of cytokine mRNA using TaqMan real-time PCR, as per the manufacturer's instructions (StepOnePlus Real-Time PCR System, Applied Biosystems-Life Technologies Australia Pty Ltd, Victoria, Australia). The selected cytokine transcripts correspond with measures of systemic inflammation that previously showed relationships with expression of zinc transporter genes (Foster et al. 2013b). Relative quantification of zinc transporter mRNA was conducted using TaqMan real-time PCR (ABI 7500 Fast Sequence Detection System; Applied Biosystems-Life Technologies Australia Pty Ltd, Victoria, Australia). Inventoried TaqMan gene expression assays, and one custom-designed assay, were obtained for ZnT1, ZnT5, ZnT6, ZnT7, ZnT8, Zip1, Zip3, Zip7, Zip10, MT-1A and MT-2A mRNA (Applied Biosystems-Life Technologies Australia Pty Ltd, Victoria, Australia). Messenger RNA expression levels for all genes were normalized to $18 S$ rRNA expression as an endogenous reference and quantified using the $\Delta C_{\mathrm{P}}$ method; fold change relative to baseline was quantified using the $\Delta \Delta C_{\mathrm{P}}$ method.

Statistical analysis

Descriptive statistics were expressed as mean \pm SD. Cytokine gene expressions and fold changes were described as mean \pm SEM. Differences in group means of baseline characteristics, cytokine expressions and fold changes were assessed by analysis of variance (ANOVA). No significant changes were observed in primary outcomes measured (glycaemia, systemic inflammatory markers or cytokine mRNA expressions) after FSO supplementation, with or without zinc. Hence, post hoc investigations using independent $t$-tests were used to compare groups categorized according to whether participants did or did not receive zinc supplementation. Post hoc analysis according to FSO supplementation was performed and confirmed no effect on cytokine gene expressions when FSO was consumed alone or in combination with zinc.

Bivariate relationships between fold changes of cytokines, zinc transporters and MT were calculated using the Pearson's correlation coefficient. Multiple regression 
models were used to explore the relationships among gene expression of $I L-1 \beta, I L-6$ and $T N F-\alpha$ and expression of all zinc transporters and MT measured, at baseline and week 12. The residuals from the regression models were checked to see whether they satisfied the assumptions of normality and homoscedasticity: the initial regressions indicated that analyses of $M T-1 A$ and $M T-2 A$ were more appropriately conducted on a log scale. Statistical analyses were carried out using SPSS (PASW) version 18. With analysis of the primary outcomes, a value of $P<0.05$ was taken to designate statistical significance. In univariate outcomes within multivariate analyses, a more conservative designation of $P<0.01$ as statistically significant was used due to the large number of statistical tests employed.

\section{Results}

\section{Participant characteristics}

Characteristics of study participants, biomarker outcomes and zinc transporter expressions have been described previously in detail (Foster et al. 2013a, b). Of the 48 enrolled participants, complete sets of cDNA samples were available from 40 participants for PBMC cytokine gene expression analysis. The age and BMI of participants were $($ mean $\pm \mathrm{SD}) \quad 65.1 \pm 8.0$ years and $28.3 \pm 5.0 \mathrm{~kg} / \mathrm{m}^{2}$, respectively. The average time since type $2 \mathrm{DM}$ diagnosis was $6.6 \pm 5.4$ years. The baseline fasting blood glucose concentration, fasting insulin and $\mathrm{HbA} 1 \mathrm{c}$ of the participants were $6.7 \pm 1.8 \mathrm{mmol} / \mathrm{L}, 67.2 \pm 36.8 \mathrm{pmol} / \mathrm{L}$ and $6.7 \pm 1.0 \%$, respectively. The mean plasma zinc concentration was $12.8 \pm 2.0 \mu \mathrm{mol} / \mathrm{L}$, which is within the reference range of $10-18 \mu \mathrm{mol} / \mathrm{L}$. The baseline serum concentrations of CRP, IL-1 $\beta$, IL- 6 and TNF- $\alpha$ were $1.7 \pm 2.2 \mathrm{mg} / \mathrm{L}, 1.0 \pm 1.9 \mathrm{pg} / \mathrm{mL}, 1.6 \pm 2.0 \mathrm{pg} / \mathrm{mL}$ and $10.3 \pm 3.5 \mathrm{pg} / \mathrm{mL}$, respectively. At baseline, there were no differences in zinc transporter and MT expressions between groups. Participant characteristics and biochemical measures at baseline are shown in Supplemental Table 1.

Markers of systemic inflammation and glycaemia

After 12 weeks of intervention, no significant changes were detected in the measures of systemic inflammation or glycaemia as a result of zinc supplementation. The mean plasma zinc concentration was significantly higher in the zinc-supplemented group when compared to those without zinc treatment after 4 weeks $(P<0.05$; Supplemental Fig. 1) and remained significantly higher at weeks 8 and 12. There were no significant changes observed in zinc transporter and MT mRNA expressions between treatment groups [reported previously in (Foster et al. 2013b)].
Table 1 Relative mRNA expression and fold changes of $I L-1 \beta, I L-6$ and $T N F-\alpha$ in PBMC (mean \pm SEM)

\begin{tabular}{lllll}
\hline & $\begin{array}{l}\text { Placebo } \\
(n=10)\end{array}$ & $\begin{array}{l}\text { FSO } \\
(n=10)\end{array}$ & $\begin{array}{l}\mathrm{Zn} \\
(n=11)\end{array}$ & $\begin{array}{l}\text { Zn + FSO } \\
(n=9)\end{array}$ \\
\hline$I L-1 \beta$ & & & & \\
Week 0 & $0.96 \pm 0.11$ & $1.72 \pm 0.39$ & $1.04 \pm 0.15$ & $0.91 \pm 0.14$ \\
Week 12 & $0.97 \pm 0.02$ & $1.33 \pm 0.06$ & $1.46 \pm 0.01$ & $0.98 \pm 0.06$ \\
Fold change & $1.08 \pm 0.24$ & $0.88 \pm 0.43$ & $1.51 \pm 0.30$ & $1.21 \pm 0.35$ \\
$I L-6$ & & & & \\
Week 0 & $0.10 \pm 0.12$ & $0.13 \pm 0.25$ & $0.05 \pm 0.36$ & $0.17 \pm 0.13$ \\
Week 12 & $0.08 \pm 0.02$ & $0.14 \pm 0.08$ & $0.08 \pm 0.02$ & $0.26 \pm 0.10$ \\
Fold change & $0.97 \pm 0.30$ & $1.24 \pm 0.29$ & $1.46 \pm 0.48$ & $1.38 \pm 023$ \\
TNF- $\alpha$ & & & & \\
Week 0 & $2.86 \pm 0.15$ & $3.85 \pm 0.16$ & $2.77 \pm 0.26$ & $2.89 \pm 0.18$ \\
Week 12 & $3.01 \pm 0.12$ & $3.16 \pm 0.27$ & $3.64 \pm 0.25$ & $3.09 \pm 0.23$ \\
Fold change & $1.10 \pm 0.12$ & $0.88 \pm 0.12$ & $1.38 \pm 0.16$ & $1.14 \pm 0.10$ \\
\hline
\end{tabular}

FSO flaxseed oil, IL-1 $\beta$ interleukin-1 $\beta, I L-6$, interleukin-6, TNF- $\alpha$ tumour necrosis factor- $\alpha, P B M C$ peripheral blood mononuclear cells

Relative mRNA expression expressed as copies of mRNA per $10^{6} 18 \mathrm{~S}$ rRNA

Fold change data are calculated using the $\Delta \Delta C_{\mathrm{p}}$ method and expressed on a $\log$ scale

Cytokine mRNA expression and fold change

At baseline, no differences were observed in the mRNA expression of $I L-1 \beta, I L-6$ or $T N F-\alpha$ among the intervention groups. TNF- $\alpha$ was the most highly expressed mRNA transcript of the cytokines measured, expressing at almost threefold of $I L-1 \beta$. Separated into the four treatment groups, no significant changes were found in cytokine gene expressions after 12 weeks of intervention (Table 1).

Secondary analyses were conducted with the four treatment groups differentiated according to whether participants received zinc treatment ( $\mathrm{Zn}$ group and $\mathrm{Zn}+\mathrm{FSO}$ group) or no zinc treatment (FSO group and placebo). In the zinc-treated group, mRNA expression levels at week 12 tended to be higher than baseline for $T N F-\alpha$ (per cent increase $27 \pm 10 \%$; mean $\pm \mathrm{SEM} ; \quad P=0.053), \quad I L-6$ $(42 \pm 17 \% ; \quad P=0.066)$, but not $I L-1 \beta \quad(37 \pm 16 \%$; $P=0.19)$. In those without zinc treatment, no significant differences were found between baseline and week 12 cytokine mRNA expressions. When the data were expressed as fold change, $T N F-\alpha$ was significantly higher $(P=0.037)$ as a result of zinc supplementation (Fig. 1). $I L-1 \beta$ fold change was marginally higher $(P=0.054)$ in the zinc-treated group, while no differences were observed in the fold change of $I L-6(P=0.17)$.

When separated into those groups who did receive FSO (FSO and $\mathrm{FSO}+\mathrm{Zn}$ group) or did not receive FSO (placebo and $\mathrm{Zn}$ group), $I L-1 \beta, I L-6$ and $T N F-\alpha$ gene expressions were not significantly different between week 0 and 12. Fold change of $I L-1 \beta, I L-6$ and $T N F-\alpha$ were not 


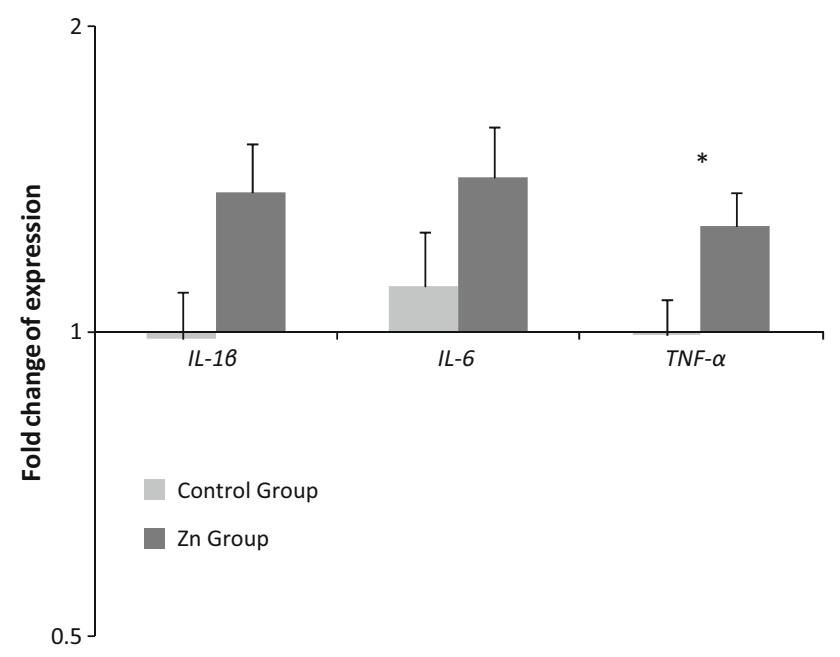

Fig. 1 Fold change of $I L-1 \beta, I L-6$ and $T N F-\alpha$ in PBMC separated by whether participants received zinc treatment $(n=20)$ or no zinc treatment $(n=20)$. Fold change data (mean \pm SEM) are calculated using the $\Delta \Delta C_{\mathrm{p}}$ method and expressed on a $\log$ scale. ${ }^{*} P<0.05$ by independent $t$-test

significantly different in the FSO group when compared to those who did not receive FSO.

Relationships among various cytokines and zinc transporters

Bivariate correlations between fold changes of individual zinc transporters and $I L-1 \beta, I L-6$ and $T N F-\alpha$ are shown in Table 2. Positive correlations between $T N F-\alpha$ fold change and fold changes of ZnT5, ZnT6, ZnT7, Zip1, Zip3, Zip7, Zip10, $M T-1 A$ and $M T-2 A$ were observed when all

Table 2 Bivariate Pearson's correlations between fold changes of cytokines, zinc transporters and metallothionein when analysed in all participants $\left(n=38^{\mathrm{a}}\right)$ and according to whether participants received participants were considered $(P<0.05)$. Similarly, $I L-1 \beta$ fold change was positively correlated to the fold change of ZnT7 ( $r=0.44, P<0.01$; Fig. 2a) and Zipl ( $r=0.42$, $P<0.01$; Fig. 2b). The treatment of zinc abolished a number of correlations between fold changes of cytokines and zinc transporters; in particular, fold changes of $I L-6$ and ZnT5 (control group, $r=0.61, P<0.01$; zinc group, $r=-0.13, \quad P>0.05$; Fig. 3), ZnT6 (control group, $r=0.49, P<0.05$; zinc group, $r=0.09, P>0.05)$ and ZnT7 (control group, $r=0.54, P<0.05$; zinc group, $r=0.32, P>0.05)$.

Using multiple regression analysis for all participants at baseline, $I L-1 \beta$ expression was predicted by expressions of all zinc transporters and MT measured $\left(r^{2}=0.495\right.$, $P=0.04$, Table 3 ), with marginally significant univariate correlations observed with Zipl $(P=0.02)$ and Zip7 $(P=0.01)$. At week 12 , overall significant multivariate relationship was maintained between expressions of $I L-1 \beta$ and zinc transporters and MT $\left(r^{2}=0.532, P=0.02\right)$ with a single significant univariate relationship found between $I L-1 \beta$ and $Z n T 7(P=0.002)$; ZnT7 expression explained $36 \%$ of the variability in $I L-1 \beta$ expression. Multiple regression analyses using expression of zinc transporters and MT as predictors of $I L-6$ or $T N F-\alpha$ expression did not show any significant relationships (data not shown).

\section{Discussion}

The key observation in the present study is an upregulation of cytokine gene expression in PBMC after zinc

(zinc, $n=20$ ) or did not receive (control, $n=18$ ) zinc supplements for 12 weeks

\begin{tabular}{|c|c|c|c|c|c|c|c|c|c|c|c|}
\hline & ZnT1 & ZnT5 & ZnT6 & ZnT7 & $Z n T 8^{\mathrm{b}}$ & Zip1 & Zip3 & Zip7 & Zip10 & $M T-1 A$ & $M T-2 A$ \\
\hline \multicolumn{12}{|l|}{$I L-1 \beta$} \\
\hline All & 0.28 & 0.28 & $0.33 *$ & $0.44 * *$ & -0.03 & $0.42 * *$ & $0.38 *$ & 0.24 & 0.14 & 0.19 & 0.36 \\
\hline Zinc & 0.33 & $0.49 *$ & 0.27 & $0.57 * *$ & -0.23 & $0.51 *$ & 0.31 & 0.27 & 0.24 & 0.10 & 0.37 \\
\hline Control & 0.31 & 0.24 & 0.42 & 0.29 & 0.04 & 0.35 & $0.70 * *$ & 0.33 & -0.06 & 0.38 & 0.32 \\
\hline \multicolumn{12}{|l|}{$I L-6$} \\
\hline All & 0.07 & 0.21 & 0.29 & $0.43 * *$ & 0.05 & 0.06 & $0.38 *$ & 0.22 & $0.42 * *$ & $0.51 * *$ & 0.14 \\
\hline Zinc & -0.08 & -0.13 & 0.09 & 0.32 & -0.13 & 0.10 & $0.46^{*}$ & 0.31 & 0.43 & $0.59 * *$ & 0.24 \\
\hline Control & 0.29 & $0.61 * *$ & $0.49 *$ & $0.54 *$ & 0.32 & 0.04 & 0.41 & 0.18 & 0.39 & 0.40 & -0.01 \\
\hline \multicolumn{12}{|l|}{$T N F-\alpha$} \\
\hline All & 0.31 & $0.39 *$ & $0.45^{* *}$ & $0.56^{* *}$ & 0.10 & $0.52 * *$ & $0.34 *$ & $0.44 * *$ & $0.33^{*}$ & $0.33 *$ & $0.60 * *$ \\
\hline Zinc & 0.31 & 0.44 & 0.41 & $0.60 * *$ & 0.11 & $0.64 * *$ & $0.49 *$ & $0.48 *$ & 0.40 & 0.28 & $0.65 * *$ \\
\hline Control & 0.42 & $0.55^{*}$ & $0.49 *$ & $0.57 *$ & -0.12 & 0.46 & 0.36 & $0.54 *$ & 0.22 & 0.43 & $0.55^{*}$ \\
\hline
\end{tabular}

$* P<0.05 ; * * P<0.01$

${ }^{\text {a }}$ Missing data for expression of zinc transporters and metallothionein for two participants

b ZnT8 mRNA expression was detected in seven participants supplemented with zinc and nine participants not supplemented with zinc 

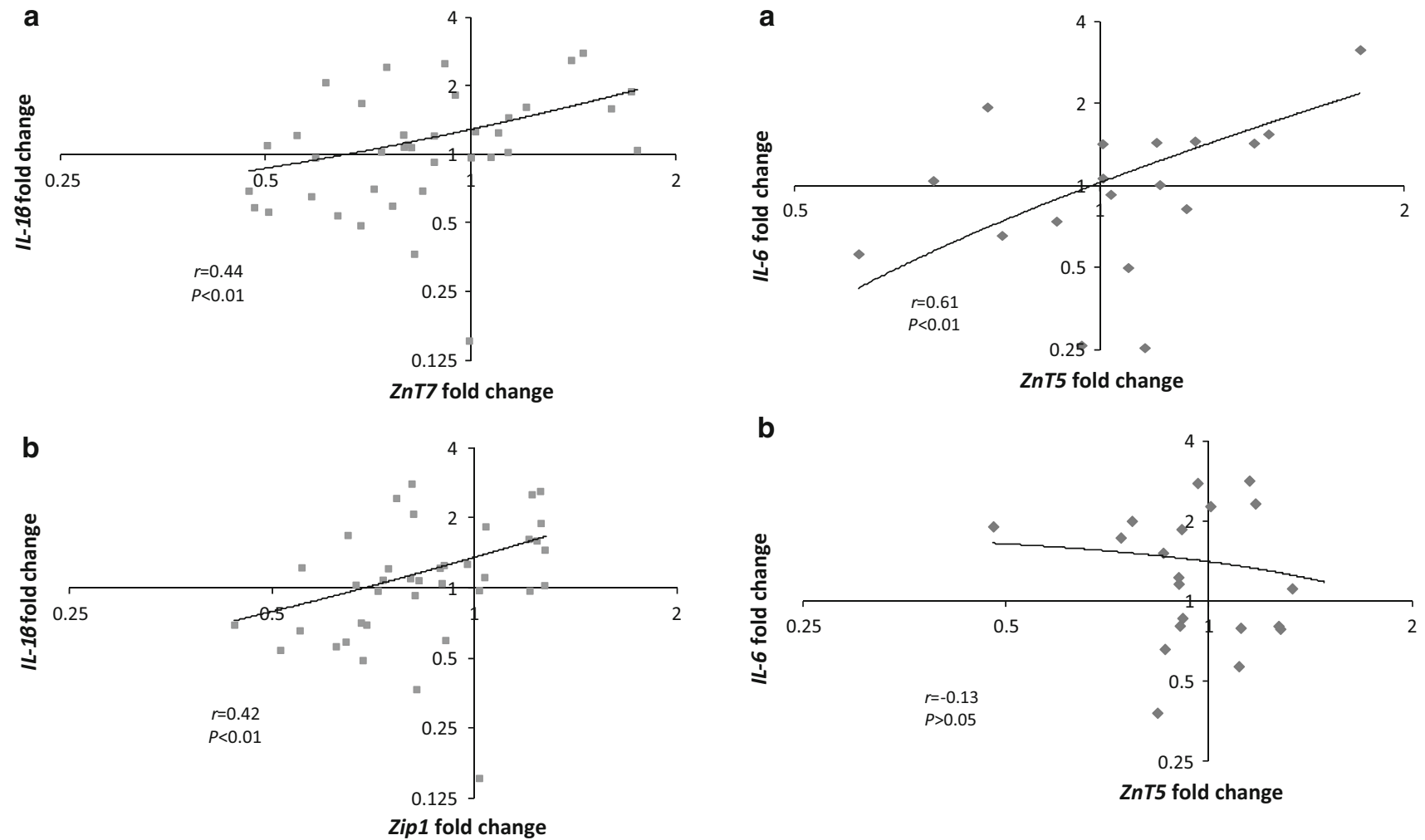

Fig. 2 Bivariate correlations between fold changes of $I L-1 \beta$ and ZnT7 (Fig. 2a), and $I L-1 \beta$ and Zipl (Fig. 2b) in all participants $(n=38)$. Fold change data are calculated using the $\Delta \Delta C_{\mathrm{p}}$ method and expressed on a log scale

supplementation in postmenopausal women with type 2 DM. Specifically, higher fold change of $T N F-\alpha$ was observed, with $I L-1 \beta$ fold change, and $I L-6$ expression tending to increase after 12 weeks of zinc supplementation. A range of bivariate relationships were found among fold changes of various cytokines and zinc transporters. In addition, zinc transporter and MT gene expressions were shown to predict the expression of $I L-1 \beta$. Collectively, the observations support a relationship between zinc and immune response in type $2 \mathrm{DM}$.

The significant upregulation observed in $T N F-\alpha$ expression after zinc supplementation in the present study is in agreement with several in vitro studies (Scuderi 1990; Wellinghausen et al. 1996; Chang et al. 2006). In cultured cells, changes in gene expression and secretion of cytokines as a result of zinc treatment have been reported. Increases in TNF- $\alpha$, IL- $1 \beta$ and IL- 6 secretion have been shown in otherwise unstimulated PBMC incubated with high levels of zinc $(\geq 100 \mu \mathrm{M})$ in the media (Wellinghausen et al. 1996; Chang et al. 2006). Similar effects were observed with zinc added at physiologically relevant levels; $T N F-\alpha$ and $I L-1 \beta$ mRNA expressions were shown to be upregulated with $30 \mu \mathrm{M}$ of zinc added to the incubating media (Chang et al. 2006). Limited data are available from

human trials on the effects of zinc supplementation on cytokine production in non-activated PBMC. The gene expressions of $T N F-\alpha$ and $I L-1 \beta$ remain unchanged in unstimulated leucocytes extracted from healthy subjects given $15 \mathrm{mg} /$ day of zinc for 4 days (Aydemir et al. 2006). The present results are novel, and to our knowledge, there are no previous reports of the effect of zinc supplementation on unstimulated cytokine expressions in postmenopausal women with type 2 DM.

Perturbation in zinc metabolism is often reported in type 2 DM (Jansen et al. 2009). The increase in the plasma zinc concentration seen in zinc-supplemented participants in the present study suggests that zinc is available for intracellular zinc replenishment in previously zinc-deficient tissues. We hypothesize that an increase in cellular zinc content in PBMC may explain these observations. The classic transcription factor, nuclear factor-kappa $\mathrm{B}(\mathrm{NF}-\mathrm{kB})$, has been proposed to be influenced by increased zinc availability. $\mathrm{NF}-\kappa \mathrm{B}$ represents the converging signalling molecule in immune cells; the propagation of Toll-like, IL-1 and TNF receptor signalling pathways is reliant on the activation of $\mathrm{NF}-\kappa \mathrm{B}$. Zinc has been proposed to enhance or inhibit NF$\kappa \mathrm{B}$ activity, dependent on the different health and/or zinc status of the studied population (Foster and Samman 2012). 
Table 3 Multiple regression models using expression of zinc transporters and metallothionein to predict $I L-1 \beta$ expression at baseline (model 1) and week 12 (model 2) for all participants

\begin{tabular}{lllllll}
\hline Factors & $\begin{array}{l}\text { Model 1 (week 0) } \\
\text { Regression } \\
\text { coefficient }\end{array}$ & $\begin{array}{l}n=38 \\
\text { Standard } \\
\text { error }\end{array}$ & $P$ value & $\begin{array}{l}\text { Model 2 (week 12) } \\
\text { Regression } \\
\text { coefficient }\end{array}$ & $\begin{array}{l}n=37 \\
\text { Standard } \\
\text { error }\end{array}$ & $P$ value \\
\hline ZnT1 & -0.02 & 0.04 & 0.58 & 0.02 & 0.07 & 0.75 \\
ZnT5 & -0.01 & 0.34 & 0.97 & -0.64 & 0.39 & 0.12 \\
ZnT6 & -0.19 & 0.23 & 0.41 & -0.09 & 0.30 & 0.78 \\
ZnT7 & 0.06 & 0.07 & 0.38 & 0.30 & 0.09 & 0.002 \\
ZnT8 & -1.38 & 2.31 & 0.55 & -4.81 & 4.55 & 0.30 \\
Zip1 & 0.18 & 0.07 & 0.02 & -0.01 & 0.09 & 0.89 \\
Zip3 & 0.11 & 0.11 & 0.35 & 0.12 & 0.14 & 0.41 \\
Zip7 & -0.34 & 0.13 & 0.01 & -0.17 & 0.26 & 0.53 \\
Zip10 & -0.01 & 0.09 & 0.91 & -0.11 & 0.12 & 0.34 \\
MT-1A & -0.11 & 0.10 & 0.28 & -0.14 & 0.16 & 0.40 \\
MT-2A & 0.05 & 0.22 & 0.82 & 0.32 & 0.44 & 0.48 \\
Overall & $r^{2}=0.495$ & & 0.04 & $r^{2}=0.532$ & & 0.02 \\
\hline
\end{tabular}

In zinc-deficient subjects, NF- $\kappa \mathrm{B}$ activation was suppressed with the consequent reduction in the cytokine production of immune cells (Prasad et al. 2006), and both of these phenomena were reversed by zinc supplementation.

Increased plasma zinc concentration, per se, may be contributing to the observed upregulation of cytokines. Higher systemic zinc concentrations may initiate intracellular signalling through an extracellular zinc receptor, GPR39. This novel zinc sensor is implicated in the regulation of cell proliferation and growth (Cohen et al. 2014), through modulation of the extracellular regulating kinase (ERK) $1 / 2$ and Akt pathways. Although the direct effect of GPR39 on intracellular signalling in the immune cells is unclear, it is conceivable that the activation of GPR39 by increased extracellular zinc may trigger downstream signalling eventuating in upregulation of cytokine expression. Given that a state of zinc deficiency appears to co-exist with type 2 DM (Jansen et al. 2009), the current findings after zinc supplementation may be evidence of improved immune response through increased efficiency in signalling pathways and thereby upregulation of cytokine gene expressions.

The multiple bivariate correlations found in the present study between fold changes of cytokine, zinc transporter and MT mRNA suggest coordination between fluxes of cellular zinc ions and cytokine production in PBMC, an important function of immune cells. These findings support previous observations of a relationship between zinc transporter gene expression and systemic inflammatory biomarkers in humans (Foster et al. 2013b; Noh et al. 2014). Systemic inflammatory biomarkers, such as CRP, IL- 6 and TNF- $\alpha$, were able to predict the gene expressions of zinc transporter and MT in PBMC; in particular, increases in systemic IL-6 concentration were related to increases in the expression of a cluster of zinc transporters, ZnT5, ZnT7, Zip1, Zip7 and Zip10, which are responsible largely for the uptake of extracellular zinc and transport of zinc into intracellular organelles and secretory pathways in PBMC. Considering that inflammatory biomarkers in the systemic circulation are the cumulative secretion of many peripheral tissues, the present study is unique in elucidating the relationship between zinc transporter, MT and cytokine gene expression within PBMC.

At both baseline and week $12, I L-1 \beta$ gene expression was predicted by zinc transporter and MT gene expressions. Furthermore, $I L-1 \beta$ expression was strongly predicted by the gene expressions of Zip1 and Zip7 at baseline and $Z n T 7$ at week 12 in univariate analyses. Considering their proposed localization within the cell (Huang and Tepaamorndech 2013; Jeong and Eide 2013), these zinc transporters are likely to be involved in either increasing cytoplasmic zinc concentration or the transport of zinc into the golgi apparatus. Taken together with the previously observed relationships between systemic IL- 6 with gene expression of ZnT7, Zipl and Zip7 (Foster et al. 2013b), we hypothesize that systemic cytokines have a regulatory control of zinc transporter transcription which influences the flux of zinc into the secretory pathway, thereby assisting in immune cell function such as $I L-1 \beta$ transcription. This hypothesis is supported by recent findings that suggest external stimuli such as systemic cytokines may regulate zinc transporter expression through the JAKSTAT pathways (Miyai et al. 2014). In particular, cytokines were found to induce the expression of Zip10, thereby providing an influx of intracellular zinc. The uptake of zinc via this particular zinc transporter appears to be critical for the survival, function and signalling of B-lymphocytes (Hojyo et al. 2014). In a similar vein, we have previously reported an association between Zip10 gene expression and glycaemic control (Foster et al. 2014), whereby the change in fasting glucose was correlated with the change in Zip10 expression. These observations 
Fig. 4 Potential zinc-mediated pathways by which external stimuli (such as systemic glucose, cytokines and zinc) may act on cellular function. External stimuli such as systemic IL-6, glucose and extracellular zinc bind to respective receptors on the plasma membrane (1). This triggers intracellular signalling events (ERK 1/2, Akt and JAK/ STAT pathways; 2), resulting in modulation of zinc transporters and MT gene expressions (3). Changes in intracellular zinc concentration can influence NF$\kappa \mathrm{B}$ activation (4) and subsequent expression of cytokines, such as TNF- $\alpha$ and IL-1 $\beta$ (5). Eventual cytokine secretions may be related to the expression of zinc transporters implicated in the secretory pathway (6)

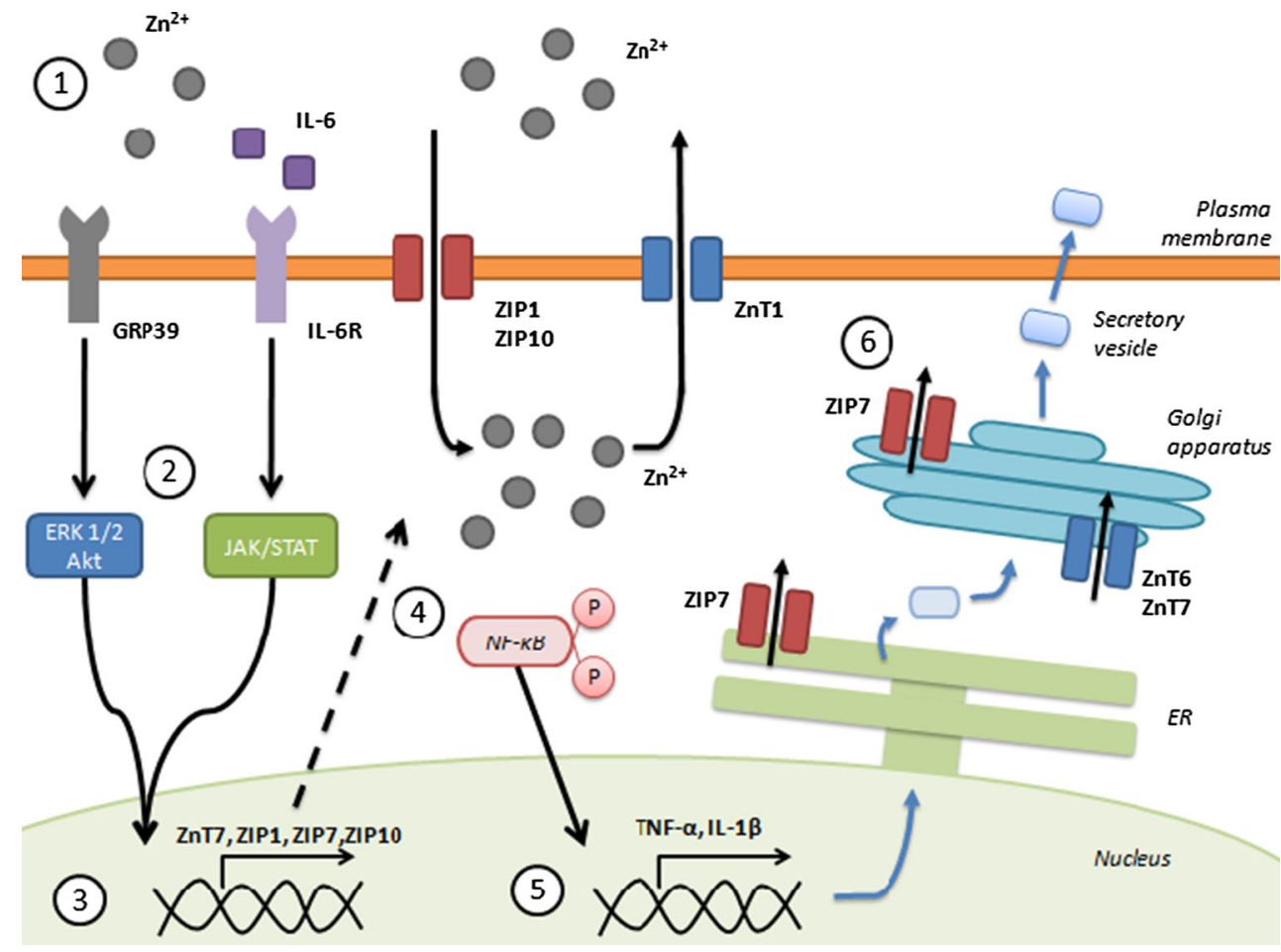

highlight the range of external stimuli that can influence zinc flux and function in the cell. Figure 4 shows the hypothesized interaction between external stimuli, zinc transport and cellular function.

The PBMC transcriptome has been shown to be reflective of gene expressions in metabolically active tissues, such as liver (Powell and Kroon 1994) and muscle (Rudkowska et al. 2011), both of which play a role in glucose and zinc homeostasis. Furthermore, modifications in dietary macronutrients and antioxidants seem to effect changes in the PBMC transcriptome (de Mello et al. 2012), hence presenting it as a promising target tissue in nutrigenomic studies. From the current study, it is evident that zinc supplementation impacts on the gene expression of cytokines and affects the relationships between cytokine and zinc transporter transcription in PBMC. In particular, the relationships between fold change of $I L-6$ with $Z n T 5$, ZnT6 and ZnT7 fold changes in the control group were not observed in the zinc-treated group. Whether zinc supplementation will elicit similar effects in cell types other than PBMC, such as those of the pancreas and other metabolically active tissues in type $2 \mathrm{DM}$, requires further investigation.

The efficacy of zinc supplementation in the management of type $2 \mathrm{DM}$ has been investigated recently. Meta-analyses have shown that zinc supplementation in type $2 \mathrm{DM}$ results in lower fasting glucose (Capdor et al. 2013) and increased high density lipoprotein concentrations (Foster et al. 2010). In the current study, zinc supplementation upregulates gene expression of cytokines in PBMC despite no changes being observed in systemic levels of the corresponding inflammatory markers (Foster et al. 2013b). Differences in the derivation of samples may provide an explanation in the discrepancy of results. In PBMC, the measure of cytokine gene expressions is upstream of cytokine secretion. Regulatory processes at post-transcriptional, translational and post-translational levels are required prior to eventual secretion of cytokines into the systemic circulation. In addition, the systemic cytokine concentration is reflective not only of PBMC cytokine secretion but from a variety of sources, such as liver, muscle and adipose tissue. Hence, the effect of increased cytokine gene expressions in PBMC remains uncertain in the context of the whole-body inflammatory status in type $2 \mathrm{DM}$.

The current study presents preliminary data on the effects of zinc supplementation on the expression of cytokines and their relationships with zinc transporter gene expressions in type $2 \mathrm{DM}$. The present observations are novel in that they provide evidence of interactions between zinc transport and cytokine transcription in PBMC, which add to our previous reports (Foster et al. 2013a, b). The study is limited by the lack of proteomic and intracellular zinc analyses. The ultimate function and activity of transcribed genes are influenced by a number of post-transcriptional regulatory processes, and further research is needed to explore the multiple possible sub-cellular localization sites of zinc transporter proteins which are influenced by the host's zinc status (Lichten and Cousins 2009). 
In summary, the current study presents preliminary evidence that zinc supplementation increases $T N F-\alpha$ gene expression in postmenopausal women with type $2 \mathrm{DM}$. The multiple relationships found between gene expression and fold change of zinc transporters, MT and cytokines suggest close interactions between zinc homeostasis and inflammation.

Acknowledgments Financial support obtained from The Medical Advances Without Animals (MAWA) Trust; and Sydnovate, The University of Sydney. The funding bodies did not play any role in designing the study; in the collection, analysis and interpretation of data; in the writing of the manuscript; or in the decision to submit the manuscript for publication.

Conflict of interest Anna Chu, Meika Foster, Dale Hancock, Kim Bell-Anderson, Peter Petocz, and Samir Samman declare that they have no conflict of interest.

\section{References}

Aydemir TB, Blanchard RK, Cousins RJ (2006) Zinc supplementation of young men alters metallothionein, zinc transporter, and cytokine gene expression in leukocyte populations. Proc Natl Acad Sci USA 103:1699-1704. doi:10.1073/pnas.0510407103

Babula P, Masarik M, Adam V et al (2012) Mammalian metallothioneins: properties and functions. Metallomics 4:739-750. doi: $10.1039 / \mathrm{c} 2 \mathrm{mt} 20081 \mathrm{c}$

Banerjee M, Saxena M (2012) Interleukin-1 (IL-1) family of cytokines: role in type 2 diabetes. Clin Chim Acta 413:1163-1170. doi:10.1016/j.cca.2012.03.021

Bao B, Prasad A (2010) Zinc decreases C-reactive protein, lipid peroxidation, and inflammatory cytokines in elderly subjects: a potential implication of zinc as an atheroprotective agent. Am J Clin Nutr 91:1634-1641. doi:10.3945/ajcn.2009.28836.1634

Calder PC (2006) N-3 polyunsaturated fatty acids, inflammation, and inflammatory diseases. Am J Clin Nutr 83:1505S-1519S

Capdor J, Foster M, Petocz P, Samman S (2013) Zinc and glycemic control: a meta-analysis of randomised placebo controlled supplementation trials in humans. J Trace Elem Med Biol 27:137-142. doi:10.1016/j.jtemb.2012.08.001

Chang K-L, Hung T-C, Hsieh B-S et al (2006) Zinc at pharmacologic concentrations affects cytokine expression and induces apoptosis of human peripheral blood mononuclear cells. Nutrition 22:465-474. doi:10.1016/j.nut.2005.11.009

Cnop M, Welsh N, Jonas J et al (2005) Mechanisms of pancreatic beta-cell death in type 1 and type 2 diabetes. Diabetes 54:97-107

Cohen L, Sekler I, Hershfinkel M (2014) The zinc sensing receptor, ZnR/GPR39, controls proliferation and differentiation of colonocytes and thereby tight junction formation in the colon. Cell Death Dis 5:e1307. doi:10.1038/cddis.2014.262

De Mello VDF, Kolehmanien M, Schwab U et al (2012) Gene expression of peripheral blood mononuclear cells as a tool in dietary intervention studies: what do we know so far? Mol Nutr Food Res 56:1160-1172. doi:10.1002/mnfr.201100685

Donath MY, Shoelson SE (2011) Type 2 diabetes as an inflammatory disease. Nat Rev Immunol 11:98-107. doi:10.1038/nri2925

Egefjord L, Jensen JL, Bang-Berthelsen CH et al (2009) Zinc transporter gene expression is regulated by pro-inflammatory cytokines: a potential role for zinc transporters in beta-cell apoptosis? BMC Endocr Disord 9:7. doi:10.1186/1472-6823-9-7
Foster M, Samman S (2010) Zinc and redox signaling: perturbations associated with cardiovascular disease and diabetes mellitus. Antioxid Redox Signal 13:1549-1573. doi:10.1089/ars.2010. 3111

Foster M, Samman S (2012) Zinc and regulation of inflammatory cytokines: implications for cardiometabolic disease. Nutrients 4:676-694. doi:10.3390/nu4070676

Foster M, Petocz P, Samman S (2010) Effects of zinc on plasma lipoprotein cholesterol concentrations in humans: a metaanalysis of randomised controlled trials. Atherosclerosis 210:344-352. doi:10.1016/j.atherosclerosis.2009.11.038

Foster M, Hancock D, Petcoz P, Samman S (2011) Zinc transporter genes are coordinately expressed in men and women independently of dietary or plasma zinc. J Nutr 1195-1201. doi:10.3945/ jn.111.140053

Foster M, Petocz P, Caterson ID, Samman S (2013a) Effects of zinc and $\alpha$-linolenic acid supplementation on glycemia and lipidemia in women with type 2 diabetes mellitus: a randomized, doubleblind, placebo-controlled trial. J Diabetes Res Clin Metab. doi:10.7243/2050-0866-2-3

Foster M, Petocz P, Samman S (2013b) Inflammation markers predict zinc transporter gene expression in women with type 2 diabetes mellitus. J Nutr Biochem 24:1655-1661. doi:10.1016/j.jnutbio. 2013.02.006

Foster M, Chu A, Petocz P, Samman S (2014) Zinc transporter gene expression and glycemic control in post-menopausal women with type 2 diabetes mellitus. J Trace Elem Med Biol. doi:10. 1016/j.jtemb.2014.07.012 [Epub ahead of print]

Haase H, Maret W (2005) Fluctuations of cellular, available zinc modulate insulin signaling via inhibition of protein tyrosine phosphatases. J Trace Elem Med Biol 19:37-42. doi:10.1016/j. jtemb.2005.02.004

Hennigar SR, Kelleher SL (2012) Zinc networks: the cell-specific compartmentalization of zinc for specialized functions. Biol Chem 393:565-578. doi:10.1515/hsz-2012-0128

Hojyo S, Miyai $\mathrm{T}$, Fujishiro $\mathrm{H}$ et al (2014) Zinc transporter SLC39A10/ZIP10 controls humoral immunity by modulating B-cell receptor signal strength. Proc Natl Acad Sci USA 111:11786-11791. doi:10.1073/pnas.1323557111

Huang L (2014) Zinc and its transporters, pancreatic $\beta$-cells, and insulin metabolism. Vitam Horm 95:365-390. doi:10.1016/ B978-0-12-800174-5.00014-4

Huang L, Tepaamorndech S (2013) The SLC30 family of zinc transporters-a review of current understanding of their biological and pathophysiological roles. Mol Aspects Med 34:548-560. doi:10.1016/j.mam.2012.05.008

Huber AM, Gershoff SN (1973) Effect of zinc deficiency in rats on insulin release from the pancreas. J Nutr 103:1739-1744

Jansen J, Karges W, Rink L (2009) Zinc and diabetes-clinical links and molecular mechanisms. J Nutr Biochem 20:399-417. doi:10. 1016/j.jnutbio.2009.01.009

Jeong J, Eide DJ (2013) The SLC39 family of zinc transporters. Mol Aspects Med 34:612-619. doi:10.1016/j.mam.2012.05.011

Kelleher S, McCormick NH, Velasquez V, Lopez V (2011) Zinc in specialized secretory tissues: roles in the pancreas, prostate, and mammary gland. Adv Nutr 2:101-111. doi:10.3945/an.110. 000232

Kim AM, Tingen CM, Woodruff TK (2010) Sex bias in trials and treatment must end. Nature 465:688-689. doi:10.1038/465688a

Lichten LA, Cousins RJ (2009) Mammalian zinc transporters: nutritional and physiologic regulation. Annu Rev Nutr 29:153-176. doi:10.1146/annurev-nutr-033009-083312

Liuzzi JP, Lichten LA, Rivera S et al (2005) Interleukin-6 regulates the zinc transporter Zip14 in liver and contributes to the hypozincemia of the acute-phase response. Proc Natl Acad Sci USA 102:6843-6848. doi:10.1073/pnas.0502257102 
Miyai T, Hojyo S, Ikawa T et al (2014) Zinc transporter SLC39A10/ ZIP10 facilitates antiapoptotic signaling during early B-cell development. Proc Natl Acad Sci U S A. doi:10.1073/pnas. 1323549111

Mlinar B, Marc J, Janez A, Pfeifer M (2007) Molecular mechanisms of insulin resistance and associated diseases. Clin Chim Acta 375:20-35. doi:10.1016/j.cca.2006.07.005

Noh H, Paik HY, Kim J, Chung J (2014) The alteration of zinc transporter gene expression is associated with inflammatory markers in obese women. Biol Trace Elem Res 158:1-8. doi:10. 1007/s12011-014-9902-1

Pickup JC (2004) Inflammation and activated innate immunity in the pathogenesis of type 2 diabetes. Diabetes Care 27:813-823. doi:10.2337/diacare.27.3.813

Powell EE, Kroon PA (1994) Low density lipoprotein receptor and 3-hydroxy-3-methylglutaryl coenzyme A reductase gene expression in human mononuclear leukocytes is regulated coordinately and parallels gene expression in human liver. J Clin Invest 93:2168-2174. doi:10.1172/JCI117213

Prasad A, Beck F (2007) Zinc supplementation decreases incidence of infections in the elderly: effect of zinc on generation of cytokines and oxidative stress. Am J Clin Nutr 85:837-844

Prasad AS, Bao B, Beck FWJ, Sarkar FH (2006) Correction of interleukin-2 gene expression by in vitro zinc addition to mononuclear cells from zinc-deficient human subjects: a specific test for zinc deficiency in humans. Transl Res 148:325-333. doi:10.1016/j.trsl.2006.07.008

Rudkowska I, Raymond C, Ponton A et al (2011) Validation of the use of peripheral blood mononuclear cells as surrogate model for skeletal muscle tissue in nutrigenomic studies. OMICS 15:1-7. doi:10.1089/omi.2010.0073

Scuderi P (1990) Differential effects of copper and zinc on human peripheral blood monocyte cytokine secretion. Cell Immunol 126:391-405. doi:10.1016/0008-8749(90)90330-T

Wellinghausen N, Driessen C, Rink L (1996) Stimulation of human peripheral blood mononuclear cells by zinc and related cations. Cytokine 8:767-771. doi:10.1006/cyto.1996.0102

Zhao G, Etherton T, Martin K (2004) Dietary $\alpha$-linolenic acid reduces inflammatory and lipid cardiovascular risk factors in hypercholesterolemic men and women. J Nutr 134:2991-2997

Zhao G, Etherton TD, Martin KR et al (2007) Dietary alpha-linolenic acid inhibits proinflammatory cytokine production by peripheral blood mononuclear cells in hypercholesterolemic subjects. Am J Clin Nutr 85:385-391 